Article

\title{
Upper Extremity Muscle Activation during Bodyblade Exercises Following Six Weeks of Intervention Focusing on the Lumbopelvic-Hip Complex
}

\author{
Gretchen D. Oliver 1,†,*, Audrey J. Stone ${ }^{2, \dagger}$, Wendi H. Weimar ${ }^{3, \dagger}$, Lawrence Lemak ${ }^{4, \dagger}$, \\ Jessica K. Washington ${ }^{3, \dagger}$ and Christopher P. Dougherty ${ }^{5, \dagger}$
}

1 School of Kinesiology, Auburn University, Auburn, AL 36849, USA; E-Mail: goliver@auburn.edu

2 Heart and Vascular Institute Penn State College of Medicine, Hershey, PA 17033, USA;

E-Mail: astone1@hmc.psu.edu

3 School of Kinesiology, Auburn University, Auburn, AL 36849, USA;

E-Mail: weimawh@auburn.edu (W.H.W.); jkw0011@auburn.edu (J.K.W.)

4 Lemak Sports Medicine and Orthopedics, Birmingham, AL 35213, USA;

E-Mail: llemak@thelemakgroup.com

5 Agility Center, Orthopedics, Bentonville, AR 72712, USA; E-Mail: cptd12@gmail.com

$\dagger$ These authors contributed equally to this work.

* Author to whom correspondence should be addressed; E-Mail: goliver@auburn.edu;

Tel.: +1-334-844-1497; Fax: +1-334-844-1467.

Academic Editor: Eling de Bruin

Received: 30 May 2015 / Accepted: 3 August 2015 / Published: 6 August 2015

\begin{abstract}
The current study examined the effects of a six-week lumbopelvic-hip complex (LPHC) strengthening program on muscle activation when performing the shoulder dump exercise. The shoulder dump is a total body exercise that includes overhead arm movements. A pre-post test with a control group was implemented. Twenty-seven graduate students ( $23.5 \pm 1.34 \mathrm{yr} ; 174.4 \pm 11.0 \mathrm{~cm} ; 76.6 \pm 16.9 \mathrm{~kg}$ ) participated. Participants were randomly assigned to a control or intervention group where the intervention group performed LPHC exercises. Two-way analysis of variance (ANOVA) revealed a significant increase in muscle activation of the serratus anterior from pre- and post-LPHC intervention, $(t=6.5, p<0.001)$. As presented by these data, incorporation of LPHC exercises may assist in facilitating greater activation in some of the scapula-stabilizing musculature that has traditionally been
\end{abstract}


rehabilitated in prone or side-lying positions. It is, therefore, suggested that LPHC exercises be incorporated in a regular shoulder rehabilitation program.

Keywords: core; electromyography; functional shoulder rehabilitation; kinetic chain

\section{Introduction}

Often, shoulder rehabilitations focus on symptom resolution while restoration of total body function tends to be overlooked [1-4]. Kibler, et al. [4,5] emphasize the importance of a shoulder rehabilitation that addresses dysfunction at the proximal end of the kinetic chain in an attempt to facilitate efficient movement at the distal end. The kinetic chain has been described to have several functions, three of which are: (1) providing a proximal stable base for distal arm mobility [6], (2) maximizing force development from the lower extremity in an upward direction, through the lumbopelvic-hip complex (LPHC), and eventually to the hand [7-9], and (3) producing torques to decrease deceleration forces [10-12]. Thus, reiterating the commonly-accepted notion that optimal shoulder function relies on the kinetic chain [13-15].

The LPHC is the central link connecting the upper extremity to the lower extremity and is often referred to as the core. Additionally, it is the musculature of the LPHC that is active through the feed-forward mechanism for all extremity movements, indicating that LPHC musculature activation occurs in anticipation of limb movements [16,17]. It is the LPHC, therefore, that assists the body, functioning as a kinetic chain and facilitating efficient movement to meet the demands of the desired task [16,17].

The kinetic chain approach to dynamic upper extremity movement patterns focuses on glenohumeral motion, first through scapular control and then through hip stability [1]. It is the pelvis, essentially, providing a platform for the scapula which, in turn, provides a stable platform for the shoulder. Thus, it is accepted that scapula control is coordinated through a stable base of support from the trunk and pelvis $[1,18]$, essentially allowing the musculature of the lumbopelvic-hip complex to initiate shoulder movement.

It has previously been established that implementing the Bodyblade (Mad Dogg Athletics, Venice, CA, USA) into a total body shoulder exercise allows for effective activation of not only the scapular stabilizing muscles, but also LPHC muscles $[18,19]$. As shoulder rehabilitation has shifted to more of a total body approach, exercises known to activate the musculature of the LPHC could be performed simultaneously with shoulder exercises [20]. If the LPHC is activated in an attempt to stabilize the pelvis, then the musculature that stabilizes the scapula will be more efficient due to the stable base of support of the pelvis. Therefore, it was the purpose of this study to determine if upper extremity muscle activation changes following a six-week intervention program focusing on LPHC exercises. It was hypothesized that those who were engaged in the intervention program would have greater upper extremity muscle activation during the shoulder dump than those in the control group. 


\section{Methods}

\subsection{Study Design}

A pretest-posttest controlled laboratory study with repeated measures on time was used. The dependent variable was the percent of maximum voluntary isometric contraction (\%MVIC) during the shoulder dump for each muscle (multifidus, latissimus dorsi, lower trapezius, middle trapezius, infraspinatus, serratus anterior) and the independent variables were time (pretest, posttest) and group (intervention and control).

\subsection{Participants}

Twenty-seven healthy collegiate graduate students (24 $\pm 1 \mathrm{yr} ; 174 \pm 11 \mathrm{~cm}$; $77 \pm 17 \mathrm{~kg}$ ), regardless of sex, consented to participate. "Healthy" was defined as having no history of any type of injury in the last six months, as well as currently participating in physical activity for at least $30 \mathrm{~min}$, four times a week. In addition, participants were excluded if they were either currently performing a LPHC-strengthening program or if they reported a previous history of shoulder pain. The University Institutional Review Board approved testing protocols used in the current study and, prior to participation, the approved procedures, risks, and benefits were explained to all participants. All participants consented and the rights of the participants were protected according to the guidelines of the University’s Institutional Review Board.

\subsection{Procedure}

Participants were randomly assigned to either the control or intervention group. All subjects participated in two EMG testing sessions that were separated by six weeks. During the six weeks between testing, the intervention group performed LPHC exercises. The LPHC exercises were based on a previous study validating the activation of the LPHC musculature [21]. All participants reported for preand post-testing prior to engaging in any vigorous activity for that day. Location of the dominant side multifidus, latissimus dorsi, lower trapezius, middle trapezius, infraspinatus, and serratus anterior were identified through palpation of the muscle belly. Prior to testing, the identified locations for surface electrode placement were shaved, abraded, and cleaned using standard medical alcohol swabs. Subsequent to surface preparation, adhesive 3M Red-Dot bipolar, $6 \mathrm{~cm} \mathrm{Ag/AgCl} \mathrm{diameter} \mathrm{disk-shaped,}$ surface electrodes (3M, St. Paul, MN, USA) were attached over the muscle bellies and positioned parallel to muscle fibers using previously-published standardized methods [22-24]. The selected inter-electrode distance was $25 \mathrm{~mm}$ [25].

Electromyographic data were collected via a Noraxon Myopac 1400L 8-channel amplifier (Noraxon USA, Inc., Scottsdale, AZ, USA). The signal was full-wave rectified and root mean squared at $100 \mathrm{~ms}$. Surface EMG data were sampled at a rate of $1000 \mathrm{~Hz}$. The surface EMG data were notch filtered at frequencies of 59.5 and $60.5 \mathrm{~Hz}$ [26]. An additional electrode was placed on the contralateral anterior superior iliac spine (ASIS) to serve as a reference lead for the examined muscles. Following the application of surface electrodes, manual muscles testing (MMT) techniques by Kendal, et al. [27] were used to determine steady state contraction. A certified athletic trainer (AT), trained in performing MMTs, 
conducted all MMTs to ensure reliability throughout testing. Three MMTs lasting five seconds were performed for each muscle, with the first and last second of each test removed to obtain steady state results. The MMT provided baseline maximum voluntary isometric contraction (MVIC) data to which all surface EMG were compared. The aforementioned surface EMG methodologies were performed again during the post testing.

Manual Muscle Testing

The multifidus was tested with the participant prone, arms crossed over chest and attempting to perform back extension and rotation to the opposite side while the AT resisted. The latissimus dorsi was tested with the participant prone with arm adducted, extended, and medially rotated. The AT applied resistance against the forearm, in the direction of abduction and slight flexion of the arm. To test the lower trapezius, the participant was prone with arm diagonally overhead (extended and abducted approximately $30^{\circ}$, in the scapular plane), in line with the lower fibers of the trapezius. The AT applied pressure against the forearm, in a downward direction. To test the middle trapezius, the participant was prone, shoulder abducted to $90^{\circ}$, externally rotated so that thumb was pointed upward, and arm fully extended while the AT applied pressure against the forearm in a downward direction. For the infraspinatus, the participant was prone with arm resting on the table and elbow flexed to $90^{\circ}$. Participant attempted to laterally rotate the shoulder, with elbow held at $90^{\circ}$ while the AT applied pressure in the direction of medial rotation

The serratus anterior was tested with the participant lying supine, shoulder forward flexed to $90^{\circ}$. The AT resisted force against the participant's fist as the participant attempted to protract.

Following MMTs, investigators instructed participants on the proper technique of the shoulder dump rehabilitation exercise, which was previously examined by McMullen and Uhl [1]. Participants were instructed to stand in a staggered stance with their left foot forward, left hip flexed, trunk flexed and rotated to the left, and right elbow at knee level. Participants were then instructed to shift weight to the right foot while extending and rotating the trunk to the right (Figure 1). All participants performed the shoulder dump exercise with a Bodyblade (Mad Dog Athletics, Venice, CA, USA) C × T (0.57 kg, $101.6 \mathrm{~cm})$. The $\mathrm{C} \times \mathrm{T}$ model was chosen because it was applicable for beginners and was easier to control [18]. Before the participants began the EMG testing, they were given an average of three minutes to warm-up and become familiar with the Bodyblade (Mad Dogg Athletics, Venice, CA, USA) as well as the shoulder dump exercise. Participants were instructed on proper posture through verbal cues and visual aids provided by an AT. After participants were familiar with the exercise technique, they were instructed to perform three repetitions of the shoulder dump (Figure 1). The repetitions were on a time count provided by one of the investigators. The exercise's repetition count was $5 \mathrm{~s}$ to reach the middle of the shoulder dump and then $5 \mathrm{~s}$ to reach the end of the exercise, for a total time of $10 \mathrm{~s}$. 

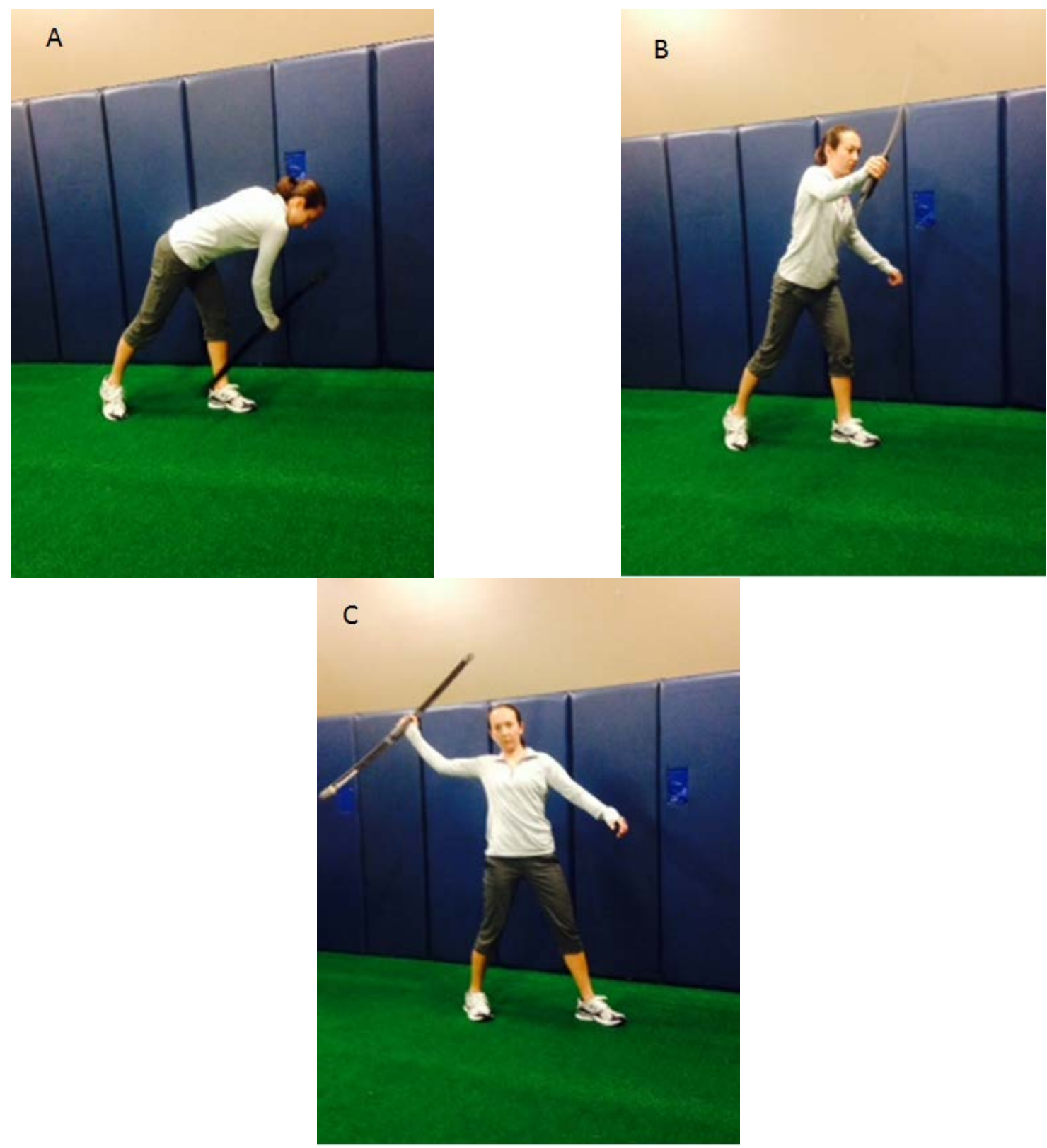

Figure 1. Shoulder Dump: (A) Start position of shoulder dump; (B) Middle of shoulder dump; (C) End position of shoulder dump.

\subsection{Intervention Program}

The intervention program consisted of a total of eight exercises (Figures 2-9), which were randomly alternated throughout the six weeks so that five exercises were performed every day of the intervention. The selection of eight consisted of isometric body weight exercises focusing on the LPHC. The participant had to choose five of the eight exercises and perform them two days a week for six weeks [28,29]. Eight exercises were given with only five being required in an attempt to allow for variation of exercise selection. Each exercise was performed for a duration of $30 \mathrm{~s}$. After completion of the exercise, $15 \mathrm{~s}$ of rest was given before the next exercise was performed. An AT familiar with the intervention exercises supervised all intervention sessions. Participants enrolled in the intervention reported to perform the intervention program under supervision at the same time and place twice a week. All participants were 
compliant with the intervention and were monitored throughout the duration by the AT. The AT monitored the participant's posture in each exercise. If the participant could not maintain the correct posture, they were instructed to reposition and continue. All participants were able to complete the exercises with minimal instructional monitoring by the AT. The control group was instructed to continue their normal physical activity of at least 30 min of physical activity, four days a week.

\section{Intervention Exercises}

Side Plank: The participant supported their body on side with weight on their forearm and lateral aspect of foot. Body remained in a straight line, pelvis was held in neutral. Position was held for 30 s (Figure 2).

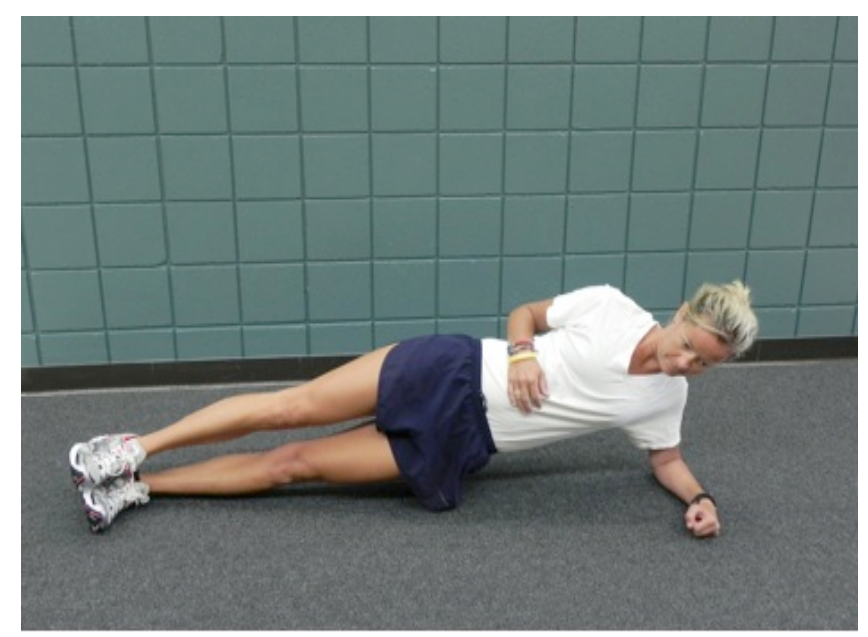

Figure 2. Side plank.

Front Plank: The participant supported their body on bilateral forearms and balls of feet. The body remained in a straight line and pelvis was held in neutral. This position was held for $30 \mathrm{~s}$ (Figure 3).

Flying Squirrel: Participant was prone with knees flexed, hips internally rotated and extended (legs off the floor), pelvis held in neutral. The shoulders were externally rotated and elbows flexed. This position was held for $30 \mathrm{~s}$ (Figure 4).

Bird Dog: The participant was on all fours and then extended contralateral arm and leg and maintained their pelvis in neutral. This position was held for $30 \mathrm{~s}$ (Figure 5).

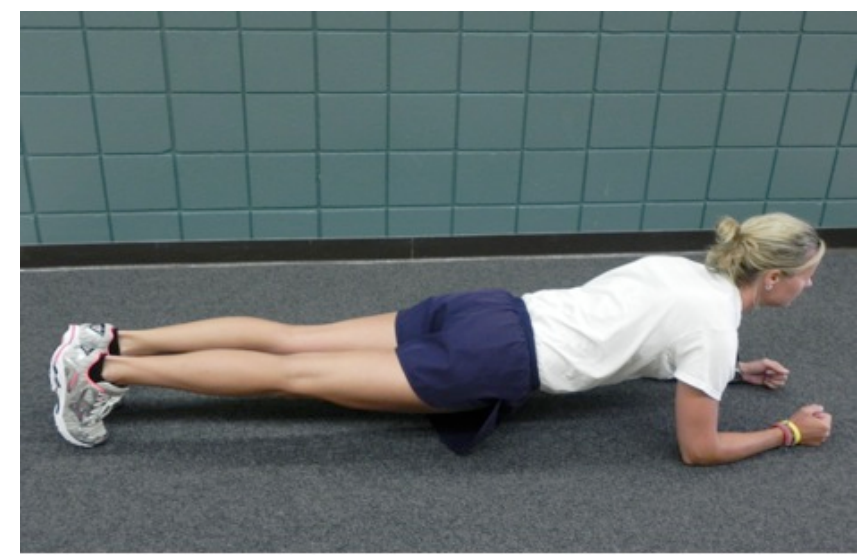

Figure 3. Front plank. 


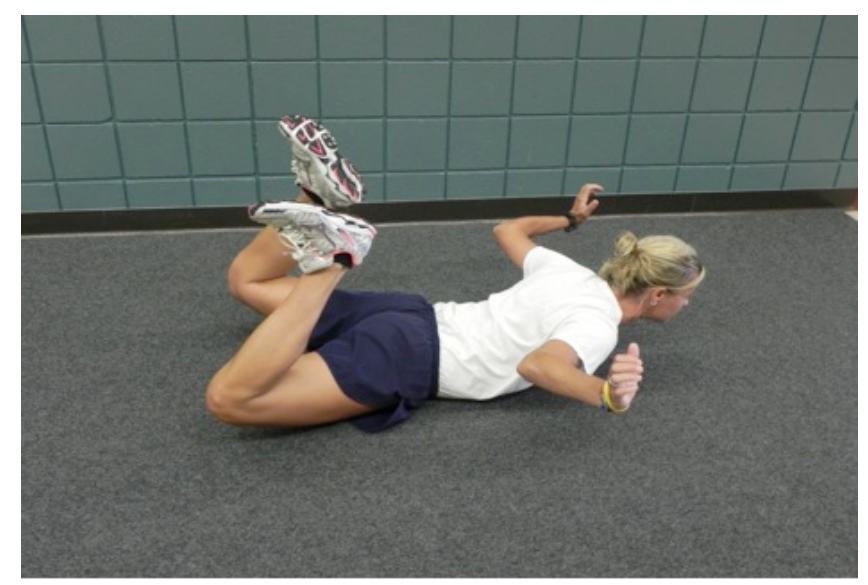

Figure 4. Flying squirrel.

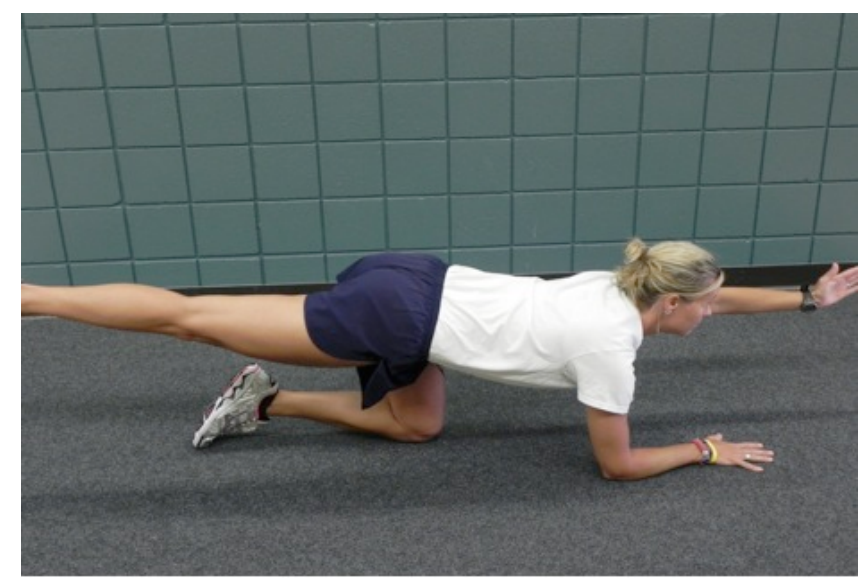

Figure 5. Bird dog.

Advanced Front Plank: Participant was in front plank position and extended contralateral arm and leg. The pelvis was held in neutral and the position was held for $30 \mathrm{~s}$ (Figure 6).

Single Leg Pelvic Tilt: The participant was supine. Participant's contralateral hip was in maximal flexion and held by participant with their hands. Ipsilateral foot was on ground. Ipsilateral foot pushed into floor to raise LPHC off floor. The participant's body remain in straight line and this position was held for $30 \mathrm{~s}$ (Figure 7).

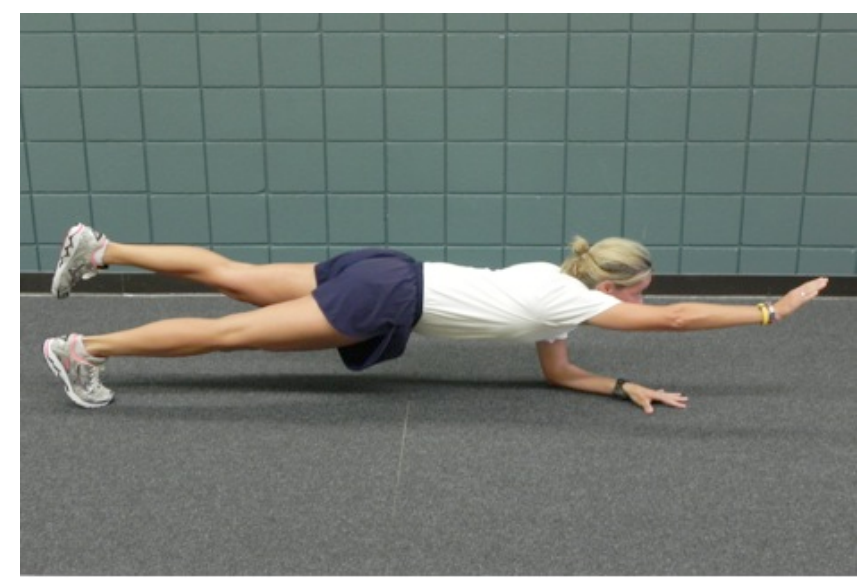

Figure 6. Advanced front plank. 


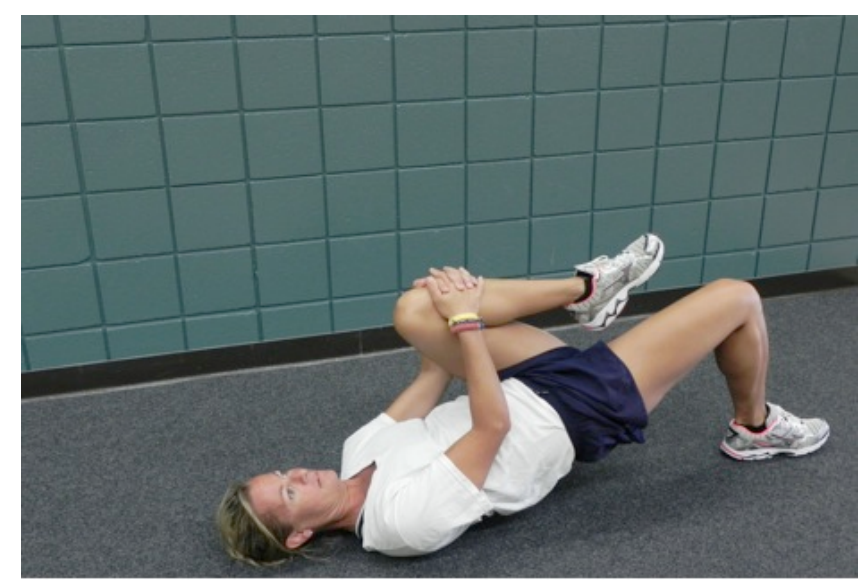

Figure 7. Single leg pelvic tilt.

Advanced Bird Dog: The participant positioned to perform the Bird Dog and then dropped into hip flexion while remaining in hip extension on the contralateral side. Position was held for $30 \mathrm{~s}$ (Figure 8). Push-Up Hold: Participant was prone with weight supported on bilateral balls of feet and palms of hands. The participant's pelvis was in neutral and body was in a straight line, this position was held for 30 s (Figure 9).

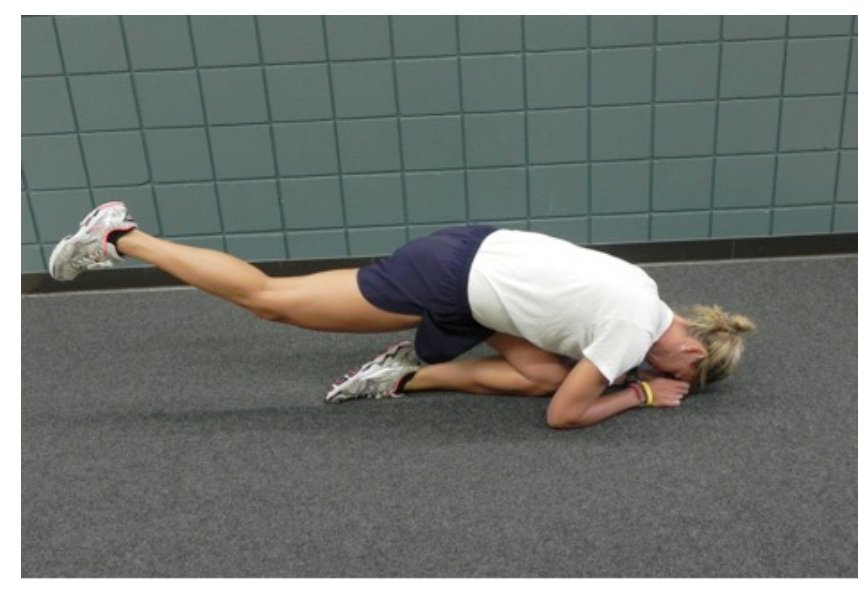

Figure 8. Advanced bird dog.

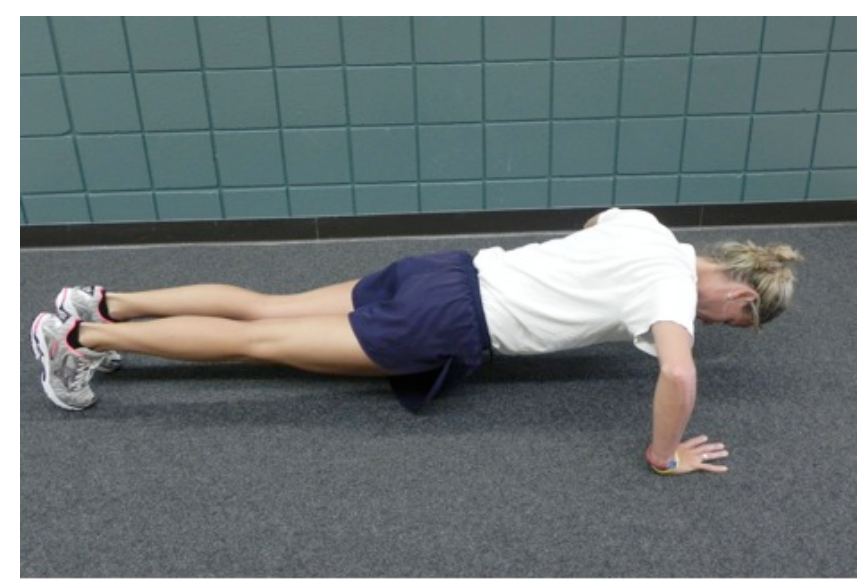

Figure 9. Push up hold. 


\subsection{Statistical Analysis}

While performing the shoulder dump, mean activation from each muscle were normalized and expressed as a percent contribution of their \%MVIC. Statistical analyses were performed using GraphPad Prism 6.0 software (GraphPad Software, Inc., La Jolla, CA, USA). Descriptive statistics were expressed by means and standard error of means (SEM). For each muscle group, a mixed model analysis of variance [ANOVA] was performed. An overall $\alpha$-level was set $a$ priori at $p \leq 0.05$. Post hoc analyses using Holm-Sidak for multiple comparisons were conducted when appropriate.

\section{Results}

Pre- and post-test \%MVICs for each muscle group were analyzed between groups. There were no significant interactions between time (pre-test vs post-test) and group (intervention vs. control) for the lower trapezius, infraspinatus, multifidus, or latissimus dorsi muscles, $p>0.05$ (Figure 10A-E). The statistical powers of the comparisons of \%MVIC within each group are shown in Table 1 . There was a significant main effect for time for the multifidus muscle (pre: $34.5 \pm 5 \% \mathrm{MVIC}$; post: $23.6 \pm 3 \%$ MVIC; $p=0.025$ ) and the latissimus dorsi muscle (pre: $25.9 \pm 4 \%$ MVIC; post: $12.9 \pm 4 \%$ MVIC; $p=0.026$ ). There was a significant interaction for the serratus anterior muscle, and post hoc analysis showed that, within the intervention group, the post-test (95.6 $\pm 13 \% \mathrm{MVIC})$ was significantly higher than the pre-test (28.3 $\pm 8 \% \mathrm{MVIC})$ (Figure 10F, $p<0.05$ ).

Table 1. Statistical power of comparisons of \%MVIC from pre- to post-test for each muscle within the control group and the intervention group.

\begin{tabular}{ccccc}
\hline & Control & \multicolumn{2}{c}{ Intervention } \\
\hline Muscle & Power & $N$ & Power & $N$ \\
Lower trapezius & 0.45 & 14 & 0.28 & 15 \\
Middle trapezius & 0.77 & 14 & 0.09 & 15 \\
Infraspinatus & 0.14 & 14 & 0.05 & 15 \\
Mutifidus & 0.84 & 14 & 0.21 & 15 \\
Latissimus dorsi & 0.98 & 14 & 0.33 & 15 \\
Serratus Anterior & 0.15 & 14 & 0.98 & 15 \\
\hline
\end{tabular}



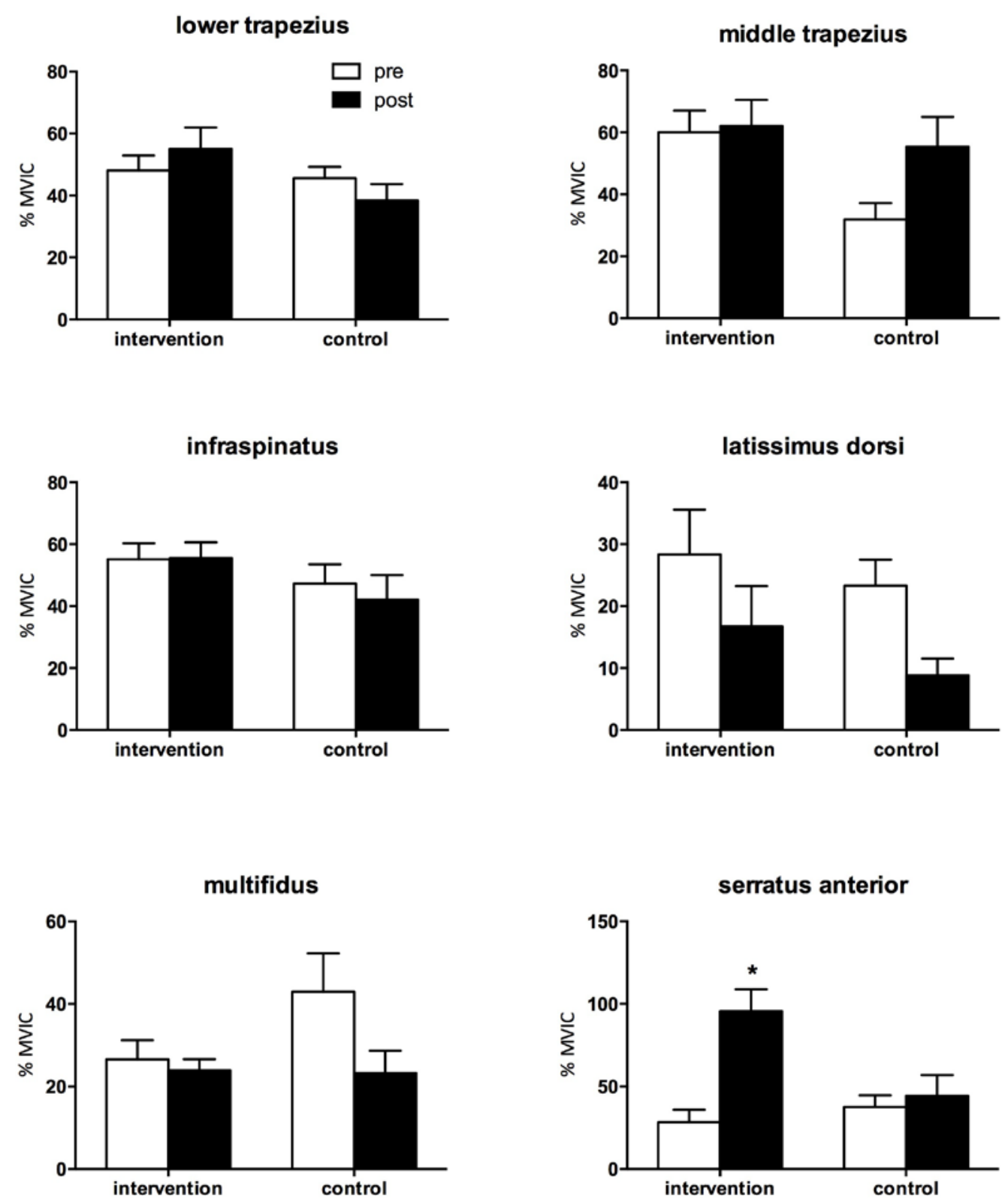

Figure 10. Percent MVIC of selected muscles presented as pre/post comparisons.

\section{Discussion}

The current study examined muscle activation of the upper extremity while performing the shoulder dump, pre- and post-intervention, as it was the aim of this study to present a LPHC intervention program to assist with achieving a total kinetic chain approach to shoulder rehabilitation. As the body functions as a kinetic chain, it works in a proximal to distal nature. Thus for efficient shoulder function, it is recommended that the proximal link, the LPHC in this case, demonstrate stability so that the distal link, the shoulder, has efficient mobility [4,5]. With the LPHC being the central link connecting the upper extremity to the lower extremity, it was hypothesized that a six-week intervention program focusing on the LPHC would result in greater upper extremity muscle activation during the shoulder dump than those in a control group. Though the only statistically significant change in muscle activation pre- and post-test was the serratus anterior, there is great clinical relevance from these results. 
The current study revealed that, when performing the shoulder dump, there was moderately strong activation (35-50\%MVIC) [30,31] of the lower trapezius, infraspinatus, and serratus anterior. This activation was consistent across the groups as well as pre- and post-testing. From a clinical standpoint, moderately strong activation has been an acceptable level for muscle strengthening [31], thus reiterating that the shoulder dump is an appropriate exercise to target the aforementioned scapular stabilizing musculature. These results are also in agreement with previously published work examining the shoulder dump exercise [18]. Additionally, it was noted that the two muscles, multifidus and latissimus dorsi, that both function in partial capacity to stabilize the pelvis, exhibited moderate to minimal (respectively) activation throughout the groups, as well as pre- and post-testing. This finding allows for clinicians to emphasis the shoulder dump, even though it is a total body activity, as more of a shoulder exercise. As for why there were decreases in activation of both the multifidus and latissimus dorsi, post-testing, is unknown. It is an intriguing find, especially with the intervention focusing on the LPHC. Thus, further investigation is needed to explain these findings.

The current study has demonstrated increased activation of scapular-stabilizing musculature, specifically the serratus anterior, in a standing posture while performing a total body functional movement pattern. Cools, et al. [32] advocates shoulder exercises where the patient is side-lying or prone as an attempt to gain the greatest lower trapezius and serratus anterior activation during the early phases of rehabilitation. However, the problem that arises is the need to have the rehabilitation mimic activities of daily living or sports-specific movement patterns. To perform the shoulder dump exercise, one has to incorporate diagonal patterns that mimic sport-specific movements. Therefore, the implementation of diagonal patterns while standing, as an attempt to engage the trunk and lower extremity, is desired in shoulder rehabilitation $[1,4,18]$.

It should be noted that the current study only examined healthy subjects and further investigation into the injured population is needed. It is unknown if there is a need to correct a LPHC problem or do we just need to make the LPHC stronger. It is known that tissue injury, as well as anatomical deficits in the lower extremity, LPHC, or scapular stabilizers can lead to overuse shoulder injury $[2,14]$. Therefore, much more attention should be placed on the function of the LPHC and its relation with not only performance but also injury prevention. Our study was able to show increased activity in scapular stabilizing muscles, specifically serratus anterior, while incorporating the LPHC in a shoulder rehab exercise.

It also should be noted that the exercise, shoulder dump, was analyzed as a complete movement and not broken down into phases. Thus with further investigation, it would be advantageous to examine the shoulder dump into phases for further insight. Additionally, the shoulder dump was the only full-body dynamic exercise examined and introduced. Moreover, other exercises could also give insight into the LPHC intervention that was introduced in this study.

In conclusion, this study was able to demonstrate that the shoulder dump exercise performed after a six-week intervention resulted in significantly greater activation of the serratus anterior. As a periscapular muscle, the serratus anterior functions as one of the scapula stabilizers during overhead movements [32-37]. The shoulder dump is a total body exercise that includes overhead arm movements. As presented by these data, incorporation of LPHC exercises may assist in facilitating greater activation in some of the scapula-stabilizing musculature that has traditionally been rehabilitated in prone or side-lying positions. 
The authors thus suggest incorporation of LPHC exercises into a regular shoulder pre-rehabilitation program in an attempt to facilitate great upper extremity muscle activation.

\section{Author Contributions}

Gretchen Oliver and Christopher Dougherty conceived and designed the experiments, Gretchen Oliver and Audrey Stone performed the experiments. Gretchen Oliver analyzed the data. Audrey Stone, Wendi Weimar, Jessica Washington, Lawrence Lemak contributed analysis tools and further analysis. Gretchen Oliver wrote the paper.

\section{Conflicts of Interest}

The authors declare no conflict of interest.

\section{References}

1. McMullen, J.; Uhl T.L. A kinetic chain approach for shoulder rehabilitation. J Athl. Train. 2000, 35, 329-337.

2. Kibler, W.B.; Press, J.; Sciascia, A. The role of core stability in athletic function. Sports Med. 2006, 36, 189-198.

3. Kibler, W.B.; Sciascia, A.D.; Uhl, T.L.; Tambay, N.; Cunningham, T. Electromyographic analysis of specific exercises for scapular control in early phases of shoulder rehabilitation. Am. J. Sports Med. 2008, 36, 1789-1798.

4. Kibler, W.B.; McMullen, J.; Uhl, T. Shoulder rehabilitation strategies, guidance and practice. Oper. Tech. Sports Med. 2012, 20, 103-112.

5. Kibler, W.B. The role of the scapula in athletic shoulder function. Am. J. Sports Med. 1998, 26, 325-337.

6. Kibler, W.B.; Kuhn, J.E.; Wilk, K.; Sciascia, A.; Moore, S.; Laudner, K.; Ellenbecker, T.; Thigpen, C.; Uhl, T. The disabled throwing shoulder: Spectrum of pathology-10year update. Arthroscopy 2013, 29, 141-161.

7. Elliott, B.C.; Marshall, R.; Noffal, G. Contributions of upper limb segment rotation during the power serve in tennis. J. Appl. Biomech. 1995, 11, 443-447.

8. Linter, D.; Noonan, T.J.; Kibler, W.B. Injury patterns and biomechanics of the athlete's shoulder. Clin. Sports Med. 2008, 27, 527-552.

9. Toyoshima, S.; Miyashita, M. Force-velocity relation in throwing. Res. Quart. 1973, 44, 86-95.

10. Putnam, C.A. Sequential motions of body segments in striking and throwing skills: Description and explanations. J. Biomech. 1993, 26, 125-135.

11. Fleisig, G.S.; Andrews, J.R.; Dillman, C.J.; Escamilla, R.F. Kinetics of baseball pitching with implications about injury mechanisms. Am. J. Sports Med. 1995, 23, 233-239.

12. Fleisig, G.S.; Barrentine, S.W.; Escamilla, R.F.; Andrews, J.R. Biomechanics of overhead throwing implications for injury. Sports Med. 1996, 21, 421-437.

13. Cordo, P.J.; Nashner, L.M. Properties of postural adjustments associated with rapid arm movements. J. Neurophysiol. 1982, 47, 287-308. 
14. Sciascia, A.; Thigpen, C.; Namdair, S.; Baldwin, K. Kinetic chain abnormalities in the athletic shoulder. Sports Med. Arthrosc. Rev. 2012, 20, 16-21.

15. Sewick, A.; Kelly, J.D.; Rubin, B. Physical examination of the overhead athlete's shoulder. Sports Med. Arthrosc. Rev. 2012, 20, 11-15.

16. Hodges, P.W.; Richardson, C.A. Relationship between limb movement speed and associated contraction of the trunk. Ergonomics 1997, 40, 1220-1230.

17. Hodges, P.W.; Richardson, C.A. Feedforward contraction of transversus abdominis is not influenced by the direction of arm movement. Exp. Brain Res. 1997, 114, 362-370.

18. Oliver, G.D.; Sola, M.; Dougherty, C.P.; Huddleston, S. Quantitative examination of upper and lower extremity muscle activation during common shoulder rehabilitation exercises using the Bodyblade. J. Strength Cond. Res. 2013, 27, 2509-2517.

19. Moreside, J.M.; Vera-Garacia, F.J.; McGill, S.M. Trunk muscle activation patterns, lumbar compressive force, and spine stability when using the Bodyblade. Phys. Ther. 2007, 87, 153-521.

20. Brumitt, J.; Dale, R.B. Integrating shoulder and core exercises when rehabilitating athletes performing overhead activities. N. Am. J. Sports Phys. Ther. 2009, 4, 132-138.

21. Oliver, G.D.; Stone, A.J.; Plummer, H. Electromyographic examination of selected muscle activation during isometric exercises. Clin. J. Sports Med. 2010, 20, 452-457.

22. Basmajian, J.V.; Deluca, C.J. Apparatus, detection, and recording techniques. In Muscles Alive, Their Functions Revealed by Electromyography, 5th ed.; Butler, J.P., Ed.; Williams and Wilkins: Baltimore, MD, USA, 1985; pp. 19-64.

23. Cram, J.R.; Kasman, G.S.; Holtz, J. (Eds.) Electrode placement. In Introduction to Surface Electromyography; Aspen Publishers: Gaithersburg, MD, USA, 1998; pp. 237-383.

24. Zipp, P. Recommendations for the standardization of lead positions in surface electromyography. Eur. J. Appl. Physiol. 1982, 50, 41-54.

25. Hintermeister, R.A.; Lange, G.A.; Schultheis, J.M.; Bey, M.J.; Hawkins, R.J. Electromyographic activity and applied load during shoulder rehabilitation exercises using elastic resistance. Am. $J$. Sports Med. 1982, 26, 210-220.

26. Oliver, G.D.; Plummer, H.A. Ground reaction forces, kinematics, and muscle activation during the windmill softball pitch. J. Sport Sci. 2011, 29, 1071-1077.

27. Kendall, F.P.; McCreary, E.K.; Provance, P.G. Muscles: Testing and Function, 4th ed.; Williams and Wilkins: Baltimore, MD, USA, 1993.

28. Oliver, G.D.; Adams-Blair, H.R.; Dougherty, C.P. Implementation of a core stability program for elementary school children. Athl. Train. Sports Health Care 2010, 2, 261-266.

29. Roling, K.; Oliver, G.D.; Dittmore, S.; Moiseichik, M. The effects of a lumpopelvic-hip strengthening intervention program on functional testing in collegiate female tennis players. Clin. Kinesiol. 2012, 66, 13-18.

30. Campbell, B.M.; Stodden, D.F.; Nixon, M.K. Lower extremity muscle activation during baseball pitching. J. Strength Cond. Res. 2010, 24, 964-971.

31. Tucker, S.; Armstrong, C.W.; Swartz, E.E.; Campbell, B.; Rankin, J.M. An electromyographic analysis of the cuff link rehabilitation device. J Sport Rehabil. 2005, 14, 124-136. 
32. Cools, A.M.; Lanszweert, F.; Notebaert, D.; Roets, A.; Soetens, B.; Cagnie, B.; Witvrouw, E.E. Rehabilitation of scapular muscle balance. Which exercise to prescribe. Am. J. Sports Med. 2007, 35, 1744-1751.

33. Cools, A.M.; Witurouw, E.E.; Declercq, G.; Danneels, L.A.; Cambier, D.C. Scapular muscle recruitment patterns: Trapezius muscle latency with and without impingement symptoms. Am. J. Sports Med. 2003, 31, 542-549.

34. Cools, A.M.; Witurouw, E.E.; Declercq, G.; Vanderstraeten, G.G.; Cambier, D.C. Evaluation of isokinetic force production and associated muscle activity in the scapular rotators during a protraction-retraction movement in overhead athletes with impingement syndrome. Br. J. Sports Med. 2004, 38, 64-68.

35. Cools, A.M.; Witurouw, E.E.; Mahieu, N.N.; Danneels, L.A. Isokinetic scapular muscle performance in overhead athletes with and without impingement symptoms. J. Athl. Train. 2005, 40, 104-110.

36. Ludewig, P.; Cook, T. Alterations in shoulder kinematics and associated muscle activity in people with symptoms of shoulder impingement. Phys. Ther. 2000, 80, 276-291.

37. Wadsworth, D.J.S.; Bullock-Saxton, J.E. Recruitment patterns of the scapular rotator muscles in freestyle swimmers with subacromial impingement. Int. J. Sports Med. 1997, 18, 618-624.

(C) 2015 by the authors; licensee MDPI, Basel, Switzerland. This article is an open access article distributed under the terms and conditions of the Creative Commons Attribution license (http://creativecommons.org/licenses/by/4.0/). 